\title{
Comprehensive analysis of a long non-coding RNA-mediated competitive endogenous RNA network in glioblastoma multiforme
}

\author{
SHENGRONG LONG and GUANGYU LI \\ Department of Neurosurgery, The First Affiliated Hospital of China Medical University, \\ Shenyang, Liaoning 110001, P.R. China
}

Received October 29, 2018; Accepted May 2, 2019

DOI: $10.3892 /$ etm.2019.7647

\begin{abstract}
The present bioinformatics study focused on glioblastoma multiforme (GBM; grade IV glioma), a common and aggressive type of primary malignant brain tumor in adults. Long non-coding RNAs (lncRNAs) function as competing endogenous RNAs (ceRNA) to regulate gene expression by interacting with microRNAs (miRNAs) in cancer. These mechanisms and phenomenon are always present but they may be deregulated or activated in cancer. In the present study, a computational method was applied to construct lncRNA-mediated ceRNA networks by integrating lncRNA and mRNA expression profiles and miRNA-mediated interactions, and functional Gene Ontology (GO) and pathway analyses were performed. From the ceRNA network, a total of 7 miRNAs, 159 lncRNAs and 31 mRNAs were obtained that were differentially expressed between GBM and adjacent tissue groups. Through survival analysis based on these RNAs from the ceRNA network, 2 mRNAs and 14 lncRNAs that had a significant impact on the survival of GBM patients were identified. Subsequently, GO and pathway analyses revealed that certain functions of the differentially expressed mRNAs were associated with processes important for the pathogenesis of GBM. The biological functions of several miRNA-mediated ceRNAs in GBM were predicted. The present study provides novel insight that may enhance the understanding of the functions of ceRNAs in GBM, as well as biomarkers for the development of therapies for GBM.
\end{abstract}

\section{Introduction}

In recent years, a vast amount of studies have demonstrated that non-coding RNAs (ncRNAs) have important biological

Correspondence to: Dr Guangyu Li, Department of Neurosurgery, The First Affiliated Hospital of China Medical University, 155 Nanjingbei Street, Shenyang, Liaoning 110001, P.R. China E-mail: liguangyu1972@sina.com

Key words: glioblastoma multiforme, long non-coding RNA, competing endogenous RNA functions. Depending on the length of the nucleic acid strands, ncRNAs may be classified as small non-coding RNA or as long non-coding RNA (lncRNA). The former may be further sub-divided into microRNA (miRNA), piwi-interacting RNA and small interfering RNA (siRNA) (1). miRNAs are a class of small non-coding RNA with lengths of 18 to 25 nucleotides. They bind to the 3'-untranslated regions (UTR) of target mRNA by complete or incomplete complementary base pairing, resulting in suppression of translation of the target gene, induction of RNA silencing or mRNA degradation, and thereby regulation of gene expression. Each miRNA is able to regulate hundreds of genes; conversely, each gene may be the target of several miRNAs. In short, miRNAs and their target genes form complex regulatory networks that participate in biological processes, including development, proliferation, differentiation and apoptosis $(2,3)$. miRNAs may be sub-divided into tumorigenic miRNA and tumor suppressor miRNA, based on their role and their target genes in tumors. Compared with miRNAs, lncRNAs have relatively long nucleotide strands, and form specific and complex secondary structures that not only provide several sites for protein binding, but also interact specifically and dynamically with DNA and RNA through the principle of complementary base pairing. In addition to being directly involved in regulation of gene expression, lncRNAs may also act as competing endogenous (ce)RNAs to compete with other RNA transcripts for the same miRNAs, thus mediating the interaction and regulation between miRNAs and mRNAs (4). The hypothesis of ceRNAs as novel regulators of gene expression was proposed recently. Serving as ceRNAs, lncRNAs, pseudogene transcripts and mRNA transcripts bind to miRNA competitively with common miRNA response elements (MREs), thereby regulating gene expression and cell function (5).

A large number of studies have confirmed that regulatory ceRNA networks of lncRNAs, miRNAs and mRNAs are associated with the pathogenesis of various cancer types, including gastric, lung and prostate cancer (6-8). However, integration analyses of lncRNA-associated ceRNA networks in glioblastoma multiforme (GBM) are rare. In the present study, the expression profiles of mRNAs, lncRNAs and miRNAs in GBM were comprehensively analyzed using cohorts from The Cancer Genome Atlas (TCGA) database and Gene Expression Omnibus (GEO), and a GBM-specific and lncRNA-associated ceRNA network was then constructed. 
All datasets of GBM and corresponding non-tumor samples from TCGA were used to establish an lncRNA-associated ceRNA network that is expected to clarify the interactions of the lncRNA-miRNA-mRNA network in GBM, as well as the molecular mechanisms involved in tumorigenesis and development of GBM.

\section{Materials and methods}

Analysis of expression profiles of mRNAs, lncRNAs and miRNAs in GBM and adjacent normal tissues. The method by Fan and Liu (9) was adopted for the design of the present study. Datasets including the quantified expression levels of mRNAs and lncRNAs in GBM were obtained from TCGA (https://cancergenome.nih.gov/). To compare lncRNA and mRNA expression signatures in GBM, all datasets that included GBM and non-tumoral brain samples were selected. The edgeR package was used to screen for differentially expressed mRNAs (DEmRNAs) and DElncRNAs in GBM and adjacent normal tissues. The expression values of lncRNAs and mRNAs were obtained by background correction and quantile normalization (10). DEmiRNAs were obtained by analyzing the dataset of GSE25631 (11) with GEO2R, a GEO online tool (https://www.ncbi.nlm.nih.gov/geo/geo2r/). The inclusion criteria were $\mid \log 2$ fold-changel $(\log 2 \mathrm{FCl})<2$ and a false discovery rate (FDR) $<0.01$ (12). Subsequently, the gplots package in $\mathrm{R}$ software was utilized to visualize meaningful RNAs with significant differences.

Construction of a ceRNA network in GBM. Interactions between DElncRNA and DEmiRNA were predicted using the miRcode database (13). mRNAs targeted by DEmiRNAs were retrieved from the databases TargetScan, miRTarBase and miRDB (14-16).

DEmRNA candidates targeted by DEmiRNAs were determined only when the mRNAs were recognized by all three databases and then overlaid with DEmRNAs. Subsequently, a lncRNA-miRNA-mRNA ceRNA network was constructed based on the DEmiRNA-DElncRNA and DEmiRNA-DEmRNA interactions, and was visualized using Cytoscape software (https://cytoscape.org/). The flow chart depicting the strategy for the IncRNA-microRNA-mRNA ceRNA network analysis is provided in Fig. 1. Pearson correlation analysis was used to assess the correlations among the expression levels of the ceRNAs.

Analysis of the influence of important RNAs in GBM with patient survival. For each specific DEmRNA and DElncRNA in the ceRNA network, GBM patients were divided into high- and low-expression groups using the median expression value as a cut-off, and the Kaplan-Meier survival curves were plotted. Subsequently, the differences in survival time between the high-expression group and the low-expression group were evaluated by using the log-rank test. The mRNAs and IncRNAs that were significantly associated with survival of GBM patients were thereby identified. $\mathrm{P}<0.05$ was considered to indicate statistical significance.

Functional enrichment analysis. Functional enrichment analysis of DEmRNAs in the ceRNA network was performed to reveal the functional significance of these mRNAs in the genesis of GBM. An online tool, the Database for Annotation, Visualization and Integrated Discovery (DAVID; http://david. ncifcrf.gov) was used for gene ontology (GO) functional enrichment analysis (17). Furthermore, Kyoto Encyclopedia of Genes and Genomes (KEGG) pathway enrichment analysis was performed by using the clusterprofiler package (18) in R software. $\mathrm{P}<0.05$ was set as the threshold for statistical significance in the GO and KEGG enrichment analysis.

\section{Results}

Cancer-specific mRNAs, IncRNAs and miRNAs in GBM. By applying the screening criteria, 2,932 DEmRNAs between GBM and normal tissues (1,341 up- and 1,591 downregulated) were identified. Furthermore, 2,291 DElncRNAs (981 upand 1,310 downregulated) and 42 DEmiRNAs (27 up- and 15 downregulated) in GBM vs. normal tissues were identified. All DElncRNAs and DEmRNAs were presented in the two dimensions of $-\log 10($ FDR) and $\mid \log F C l$ through a volcano plot. The expression levels of all of the lncRNAs and mRNAs were standardized into the averages of samples (Fig. 2).

DElncRNA-DEmiRNA interaction predicted with the miRcode database. The lncRNAs targeted by certain miRNAs were predicted by using the miRcode database. Only interactions between DElncRNAs and DEmiRNAs were selected for construction of the ceRNA network. A total of 334 interaction pairs were identified between the 159 DElncRNAs and 7 DEmiRNAs.

Prediction of DEmRNAs targeted by DEmiRNAs. The 7 DEmiRNAs identified in the abovementioned steps were inputted into the TargetScan, miRTarBase and miRDB databases to search for their target mRNAs. In all three databases, a total of 305 mRNAs were identified as targets of these DEmiRNAs. These 305 candidate mRNAs were intersected with 2,932 DEmRNA candidates predicted by miRcode with 31 DEmRNAs differentially expressed and shared as targets (Fig. 3). To emphasize the ceRNA characteristics of the network, only DEmiRNAs interacting with DEmRNAs and DElncRNAs were introduced into the ceRNA network. Finally, 7 DEmiRNAs, 31 DEmRNAs and 159 DElncRNAs were selected for construction of the ceRNA network.

ceRNA network in GBM. According to the abovementioned data and results, a lncRNA-miRNA-mRNA ceRNA network incorporating 197 molecules and 367 interactions (335 DEmiRNA-DElncRNA and 32 DEmiRNA-DEmRNA interactions) was constructed. The top eight DElncRNAs targeted by most DEmiRNAs are provided in Table I. Only five of seven DEmiRNAs with their target DEmRNAs in the ceRNA network are presented in Table II as two DEmiRNAs did not have target DEmRNAs. The network was visualized by Cytoscape and displayed in Fig. 4. The ceRNA network suggests the possibility of indirect interactions between DElncRNAs and DEmRNAs. In order to confirm this, a Pearson correlation analysis was performed, revealing a positive correlation of the expression levels of certain RNAs (Figs. 5 and 6). For 


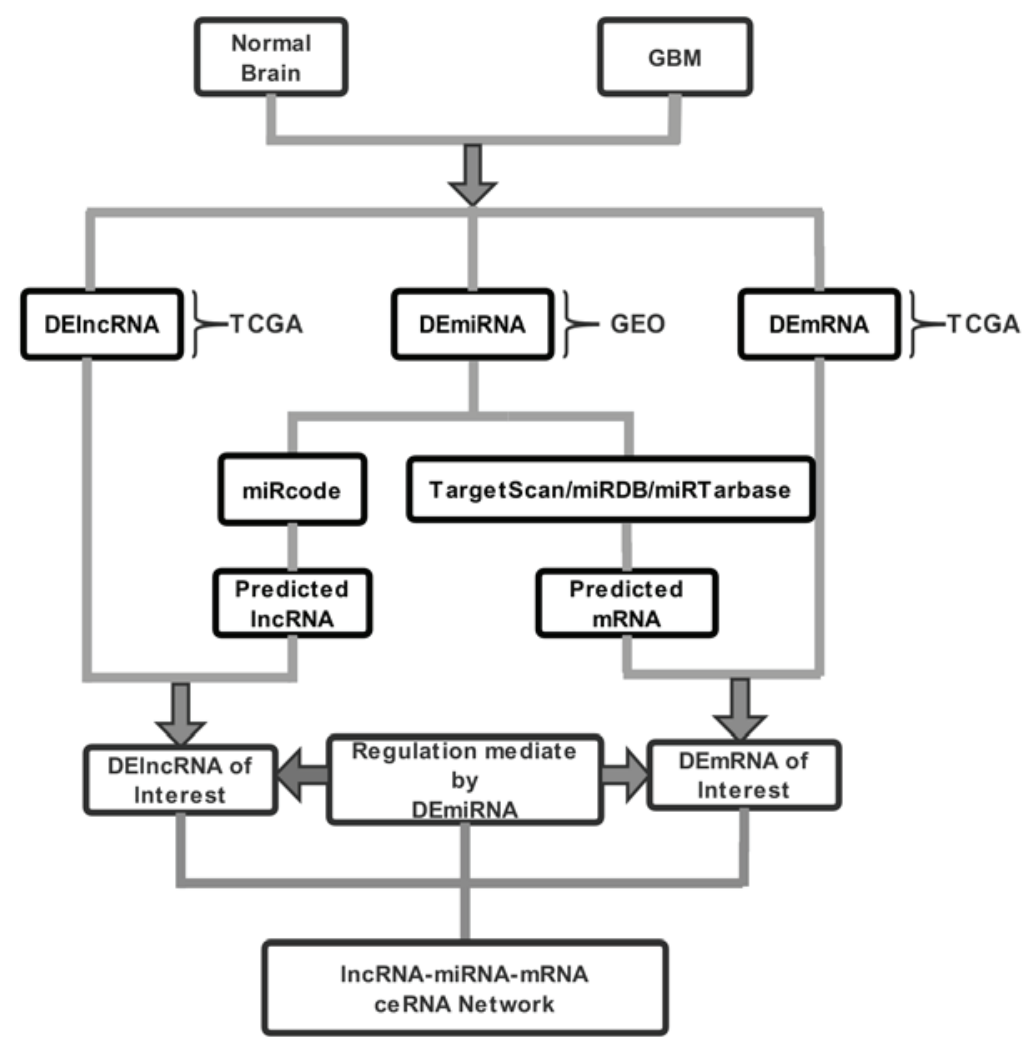

Figure 1. Flowchart of the IncRNA-miRNA-mRNA ceRNA network analysis. TCGA, The Cancer Genome Atlas; GEO, Gene Expression Omnibus; DEmiRNA, differentially expressed microRNA; GBM, glioblastoma multiforme; lncRNA, long non-coding RNA; ceRNA, competing endogenous RNA.

A

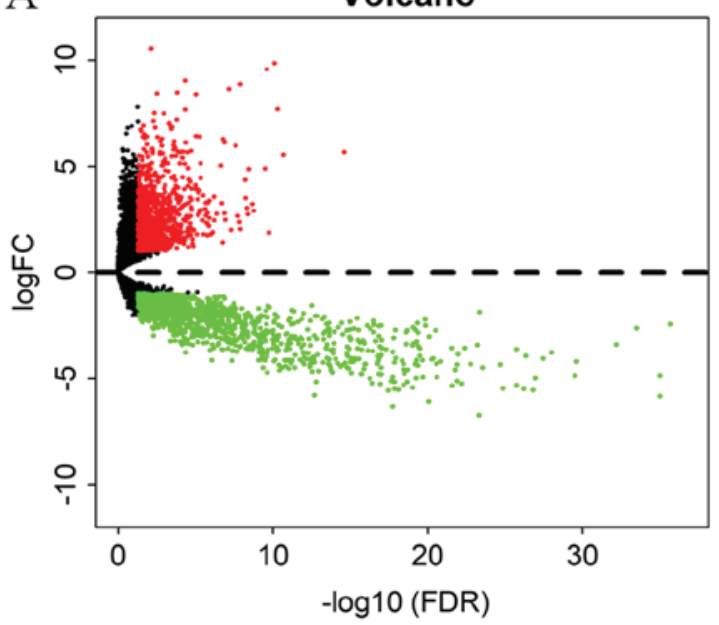

$\mathrm{B}$

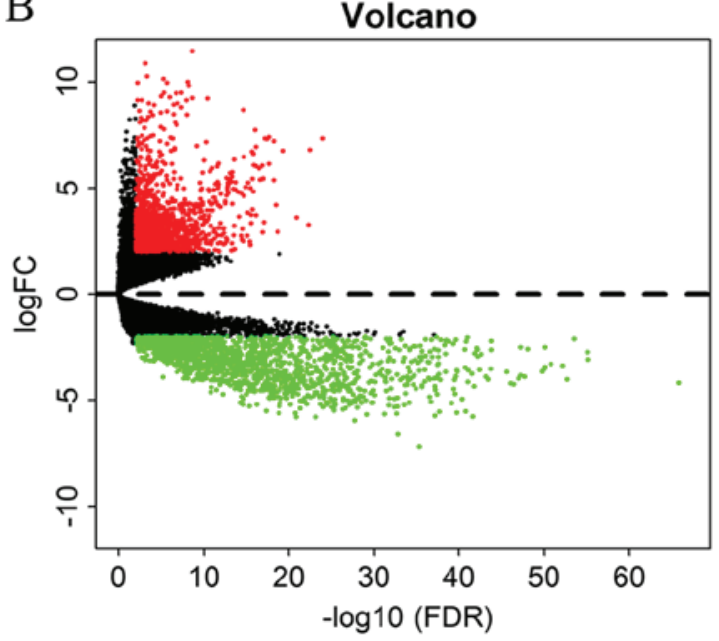

Figure 2. Volcano plot of DEmRNAs and DElncRNAs in glioblastoma multiforme. (A) DEmRNAs and (B) DElncRNAs. Red dots represent upregulated genes and green dots represent downregulated genes. DEmiRNA, differentially expressed microRNA; lncRNA, long non-coding RNA; FC, fold change; FDR, false discovery rate.

instance, LINC00336 interacted with calmodulin (CALM3), synuclein $\alpha$ (SNCA), zinc finger protein 365 (ZNF365) and family with sequence similarity 126 member B (FAM126B), which was mediated by LINC00205, and Homo sapiens (has)-miR-7 interacted with mitogen-activated protein kinase kinase kinase 10 (MAP3K10), HIV type I enhancer binding protein 2 (HIVEP2) and Rap guanine nucleotide exchange factor 2 (RARGEF2), which was mediated by hsa-miR-155 (Figs. 5 and 6).
Survival analysis. In the Kaplan-Meier analysis, a total of 14 DElncRNAs and 2 DEmRNAs were identified to be significantly associated with overall survival (Figs. 7 and 8). Among these, the expression of AC022498.1, mediator complex subunit 4 (MED4)-antisense 1 (AS1), zinc finger E-box binding homeobox 1 (ZEB1)-AS1 and zinc finger protein 197 (ZNF197)-AS1 was positively associated with survival (Fig. 7); GBM patients with higher expression levels of these RNAs in their tumor tissues tended to have a longer survival 


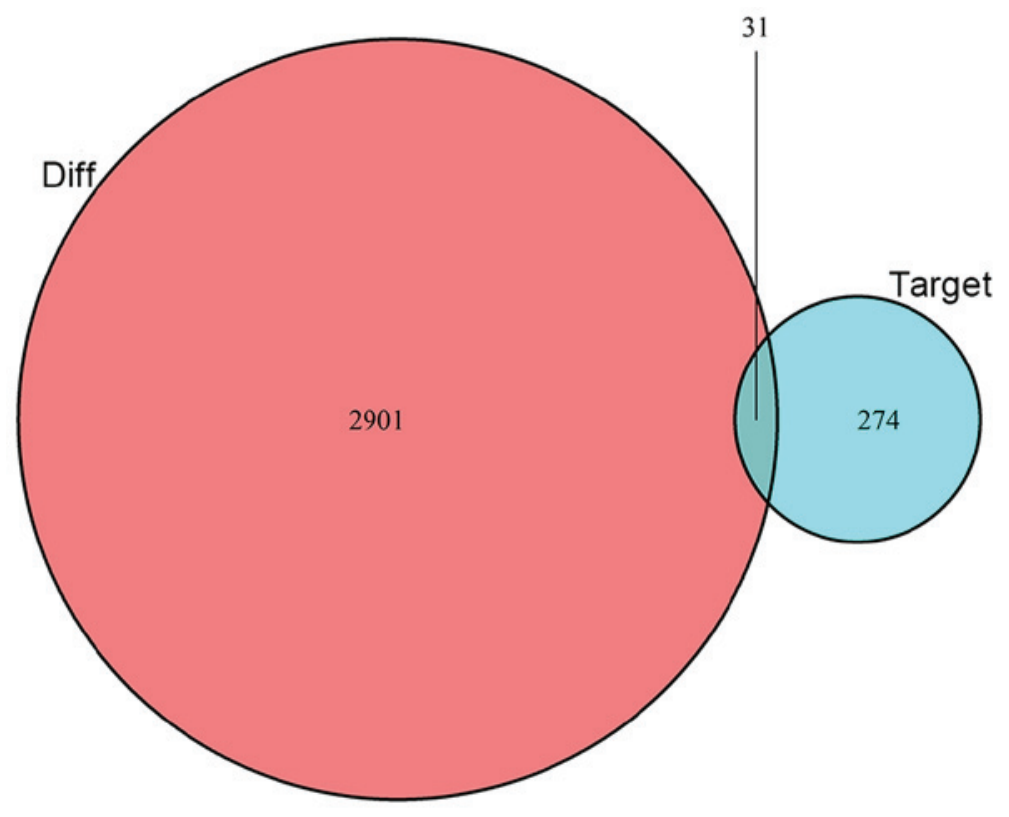

Figure 3. Venn diagram of mRNAs involved in the competing endogenous RNA regulation network. The number of differentially expressed mRNAs is represented by the red area and the blue area represents the number of targets, while the purple area in the middle represents the number of mRNAs that are differentially expressed and that are targets. Diff, differentially expressed mRNA.

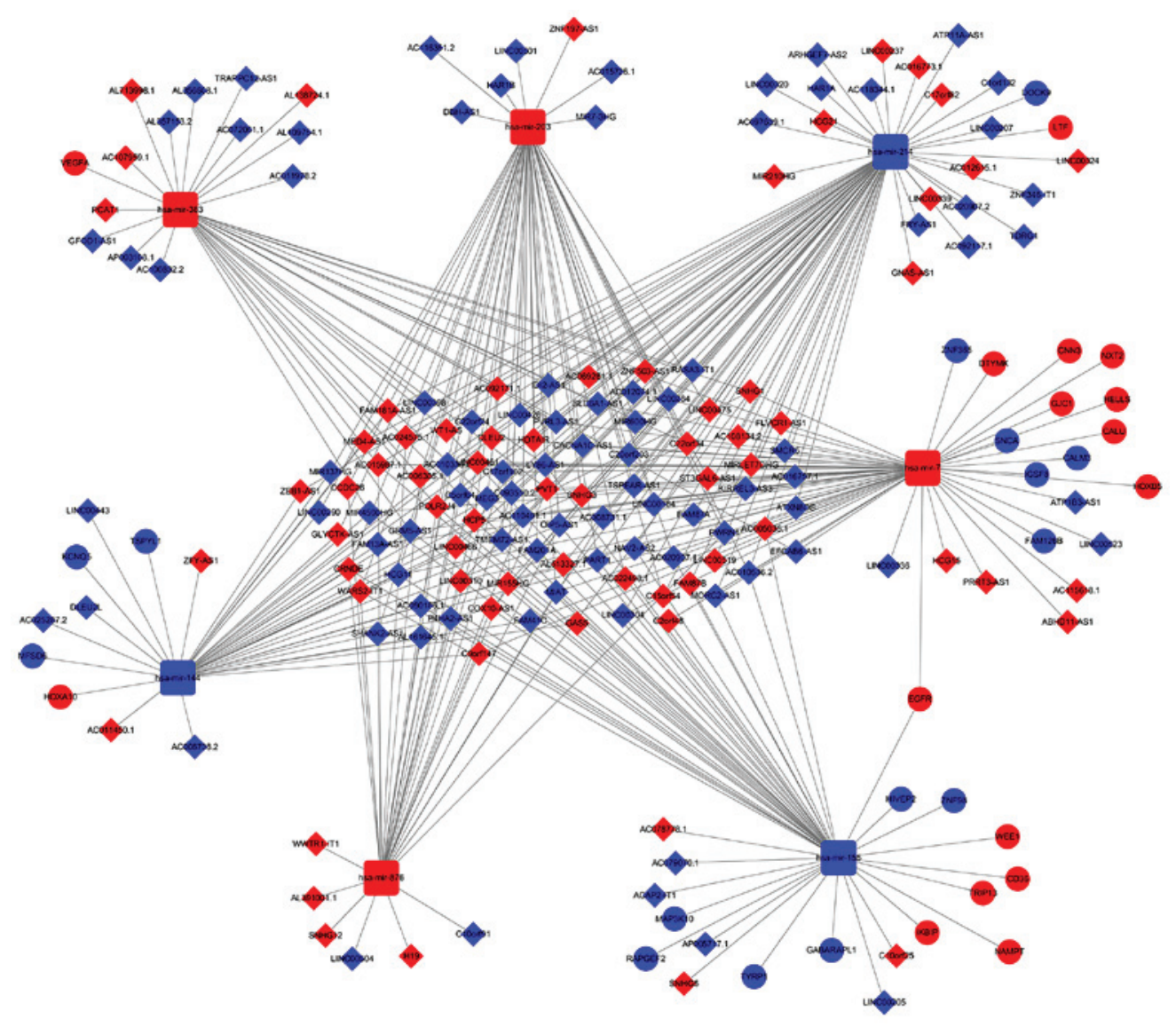

Figure 4. Competing endogenous RNA network in glioblastoma multiforme. Red circles represent upregulated DEmRNAs and blue circles represent downregulated DEmRNAs. Red squares represent upregulated DEmiRNAs and blue squares represent downregulated DEmiRNAs. Red diamonds represent upregulated DElncRNAs and blue diamonds represent downregulated DElncRNAs. DEmiRNA, differentially expressed microRNA; lncRNA, long non-coding RNA; hsa, Homo sapiens.

time compared with those with lower expression levels of these RNAs. Furthermore, the two DEmRNAs and the remaining
10 DElncRNAs were deemed risky and negatively associated with overall survival time. 
Table I. Top 8 DElncRNAs putatively targeted by most DEmiRNAs in the competing endogenous RNA network.

\begin{tabular}{ll}
\hline DElncRNAs & \multicolumn{1}{c}{ DEmiRNAs } \\
\hline HOTAIR & hsa-miR-203, hsa-miR-214 \\
H19 & hsa-miR-876 \\
WT1-AS & hsa-miR-155, hsa-miR-203, hsa-miR-383 \\
AC022498.1 & hsa-miR-155 \\
LINC00466 & hsa-miR-155, hsa-miR-214, hsa-miR-144 \\
WARS2-IT1 & hsa-miR-876, hsa-miR-144, hsa-miR-155, \\
& hsa-miR-203 \\
LINC00475 & hsa-miR-203, hsa-miR-214 \\
CRNDE & hsa-miR-876, hsa-miR-144, hsa-miR-155, \\
& hsa-miR-203
\end{tabular}

DEmiRNA, differentially expressed microRNA; lncRNA, long non-coding RNA; hsa, Homo sapiens; AS, antisense; LINC, long intergenic non-protein coding RNA; WARS2-IT1, tryptophanyl tRNA synthetase 2, mitochondrial intronic transcript 1 .

Functional enrichment analysis. Functional enrichment analysis based on DEmRNAs in ceRNA networks provided $10 \mathrm{GO}$ terms, including five terms in the category biological function, three terms in the category cellular component and two terms in the category molecular function. Certain GO terms comprised transcriptional regulation, including negative regulation and DNA template (GO:0045892) and transcriptional activation activity and binding to transcription factors of RNA polymerase II (GO:0001190) (Fig. 9). KEGG pathway enrichment analysis of all 31 DEmRNAs was also performed. One KEGG pathway, hsa04015: Rap1 signaling pathway, was identified to be significantly enriched (Fig. 10). Within this pathway, calmodulin $3(\mathrm{CaM})$ and rap guanine nucleotide exchange factor 2 (PDZ-GEF) genes were enriched significantly.

\section{Discussion}

Gliomas are central nervous system tumors with a high incidence and the highest mortality rate among brain tumors. The median survival time in patients with glioblastoma is 12-15 months (19). Tumor-associated ceRNAs regulate the occurrence and development of tumors. ceRNAs, including pseudogenes and lncRNAs, may be potential oncogenes or tumor suppressor genes involved in biological processes of the tumors.

miRNA sponges are synthetic transcripts that link several copies of MRE and clones to viral vectors. As specific inhibitors of miRNAs, they inhibit the activity of miRNAs (20). ceRNAs may be thought of as endogenous sponges; they have substantial advantages of inhibiting several MREs of various miRNAs compared to miRNA sponges. miRNA sponges and endogenous sponges of ceRNA may become targets for the RNA-based treatment of diseases including cancer in the future (21).

The theory of ceRNA will challenge certain traditional theories. For instance, mRNA requires to be translated into
Table II. DEmiRNAs with their target DEmRNAs in the competing endogenous RNA network.

DEmiRNAs DEmRNAs

$\begin{array}{ll}\text { hsa-miR-155 } & \text { HIVEP2, ZNF98, NAMPT, WEE1, TYRP1, } \\ & \text { TRIP13, IKBIP, GABARAPL1, CD36, } \\ & \text { MAP3K10, EGFR, RAPGEF2 } \\ \text { hsa-miR-7 } & \text { DTYMK, FAM126B, GJC1, ZNF365, IGSF8, } \\ & \text { NXT2, CALU, CNN3, CALM3, SNCA, } \\ & \text { HELLS, EGFR, HOXB5 } \\ \text { hsa-miR-144 } & \text { KCNQ5, HOXA10, TSPYL1, MFSD6 } \\ \text { hsa-miR-214 } & \text { LTF, DOCK9 } \\ \text { hsa-miR-383 } & \text { VEGFA } \\ \text { hsa-miR-876 } & - \\ \text { hsa-miR-203 } & -\end{array}$

DEmiRNA, differentially expressed microRNA; lncRNA, long non-coding RNA; hsa, Homo sapiens; MAP3K10, mitogen-activated protein kinase 10; RAPGEF2, Rap guanine nucleotide exchange factor 2; HIVEP2, HIV type I enhancer binding protein 2; SNCA, synuclein $\alpha$; ZNF365, zinc finger protein 365; FAM126B, family with sequence similarity 126 member B.

protein prior to exerting certain biological functions, or only affect functions of the coding region while neglecting those in the UTR (as binding sites of miRNA may appear in the UTR, the entire function of a gene may be ignored).

In the present study, large cohorts from TCGA and GEO databases were used to identify DElncRNAs, DEmRNAs and DEmiRNAs between GBM and normal tissues. A lncRNA-miRNA-mRNA ceRNA network was then built to provide an integrated view of the ceRNA-regulatory crosstalk among GBM-specific RNA transcripts. Functional enrichment analysis further revealed the GO terms and pathways associated with DEmRNAs that have a role in the development of GBM.

In the present study, 14 DElncRNAs, namely AC005035.1, AC010336.2, AC022498.1, AC108134.2, AC116351.2, C1orf132, C10orf91, DBH-AS1, HOTAIR, LINC00475, MED4-AS1, MIR210HG, ZEB1-AS1 and ZNF197-AS1, were identified to be significantly associated with the prognosis of GBM patients from the ceRNA network.

Of the 14 lncRNAs, all except AC022498.1, MED4-AS1, ZEB1-AS1 and ZNF197-AS1, may be considered risk factors, as the patients with high expression levels of these 10 markers had a shorter lifespan than those with lower levels. While little is known about the functions of these 10 DElncRNAs, the results suggest that they have a certain predictive value regarding prognosis and further study of the involvement of the 10 lncRNAs in GBM in other directions is warranted.

As the hub element of the ceRNA network, miRNA has a crucial role in the interaction among various RNA transcripts. LINC00336 interacts with CALM3, FAM126, SNCA and ZNF365 with the mediation of hsa-miR-7, while LINC00205 interacts with HIVEP2, MAP3K10 and RAPGEF2 with the mediation of hsa-miR-155. Of note, hsa-mir-7 and hsa-miR-155 have been previously reported to be involved 

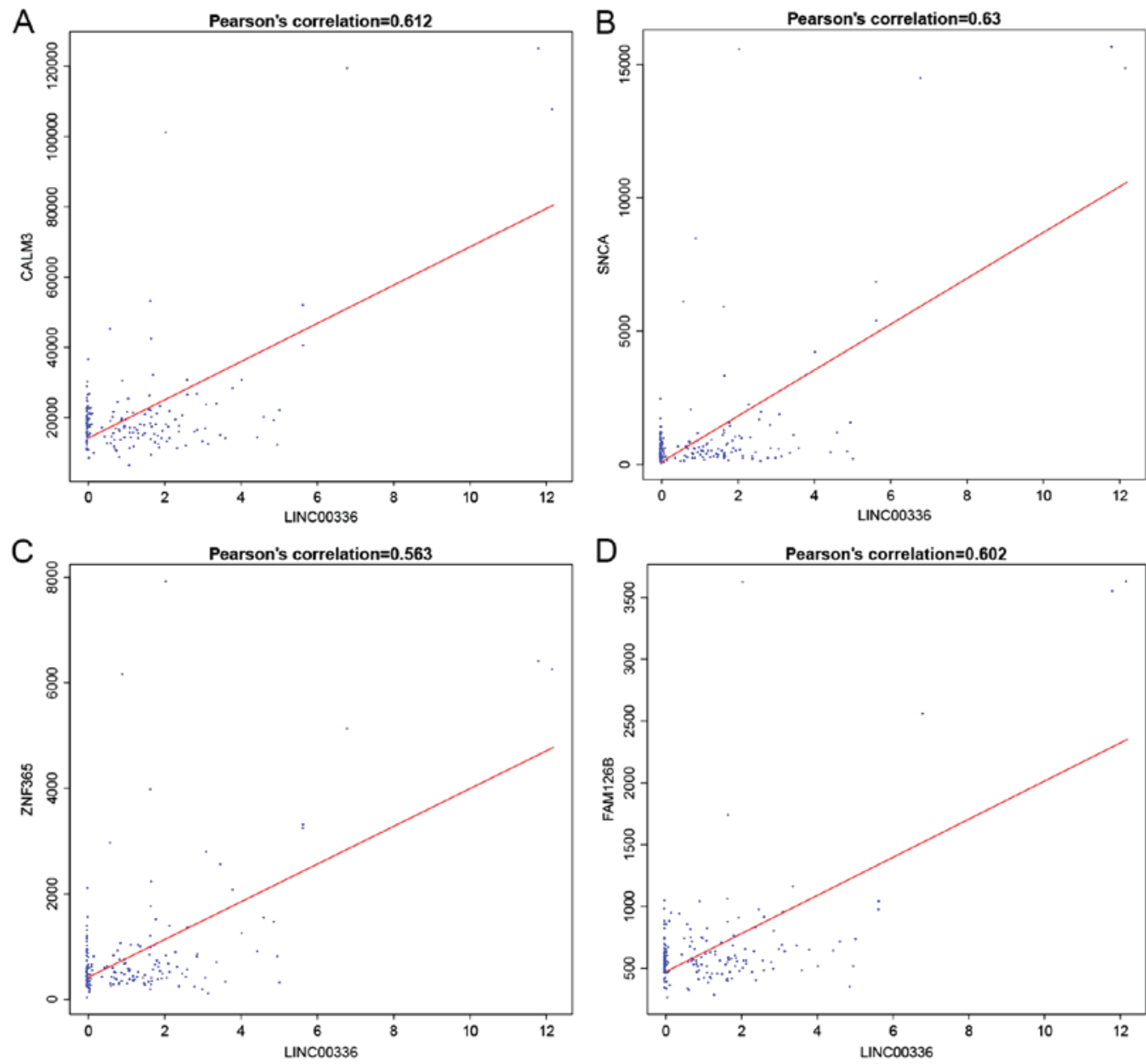

Figure 5. Pearson's correlation analysis of competing endogenous RNA expression levels for LINC00336. LINC00336 vs. (A) CALM3, (B) SNCA, (C) ZNF365 and (D) FAM126B. The red lines represent the linear model fitted by the dots in each figure. LINC00336, long intergenic non-protein coding RNA 336; CALM, calmodulin; SNCA, synuclein $\alpha$; ZNF365, zinc finger protein 365; FAM126B, family with sequence similarity 126 member B.
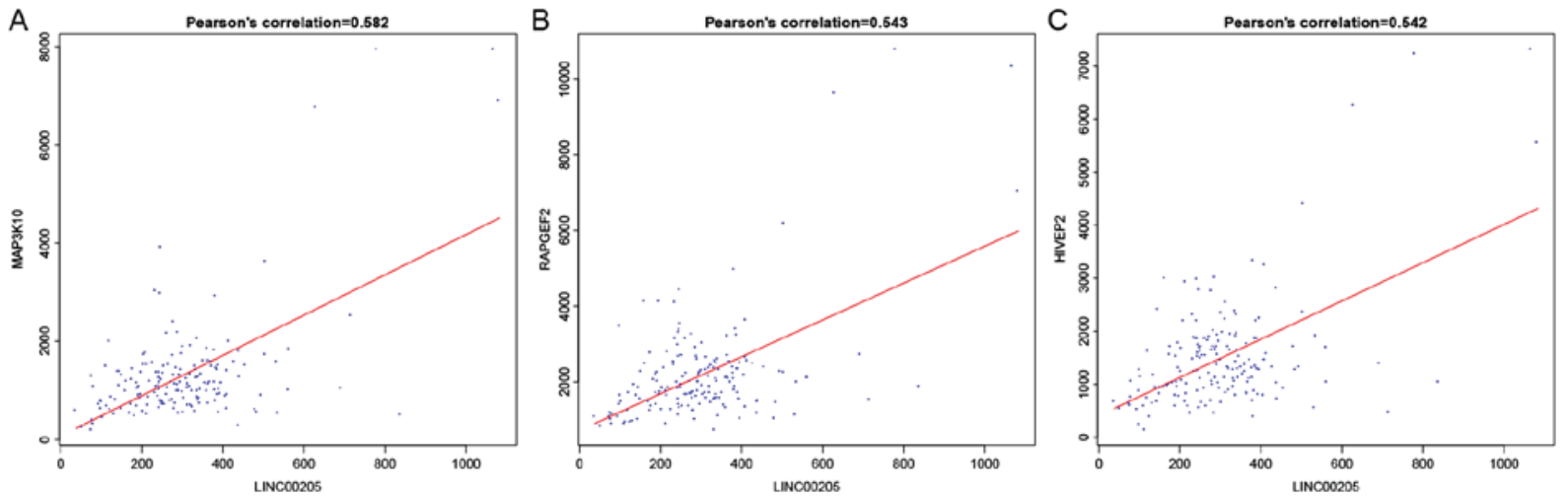

Figure 6. Pearson's correlation analysis of competing endogenous RNA expression levels for LINC00205. LINC00205 vs. (A) MAP3K10, (B) RAPGEF2 and (C) HIVEP2. The red lines represent the linear model fitted by the dots in each figure. LINC00205, long intergenic non-protein coding RNA 205; MAP3K10, mitogen-activated protein kinase kinase kinase 10; RAPGEF2, Rap guanine nucleotide exchange factor 2; HIVEP2, HIV type I enhancer binding protein 2.

in the pathogenesis of GBM $(22,23)$. All of the important DEmiRNAs had promising potential as biomarkers for survival prediction in GBM. Hsa-miR-155 has been reported as a key miRNA in breast cancer (24). As for hsa-miR-7, circular RNA U2 small nuclear RNA auxiliary factor 1 was indicated to promote human glioma by de-repressing neuro-oncological ventral antigen 2 by sponging hsa-miR-7-5p (25). While in the study by Cao et al (26), the target miRNAs were obtained only by predicting miRNA-lncRNA interactions through databases, the present study identified DEmiRNAs by using GEO2R to analyze the dataset GSE25631 on GBM.

mRNA constitutes another important part of the ceRNA network that directly targets miRNAs or interacts indirectly with IncRNAs mediated by miRNAs. Comparable 

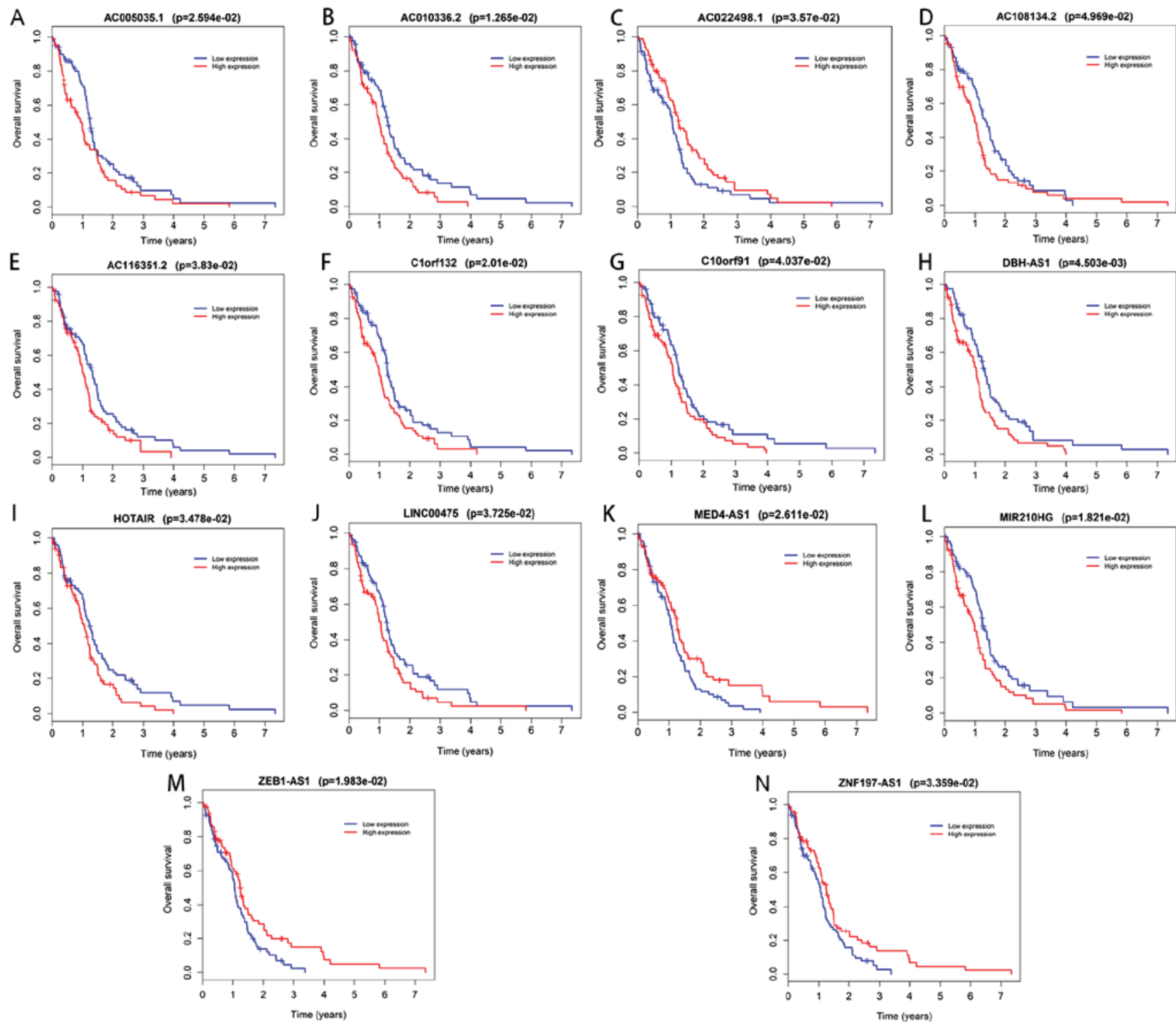

Figure 7. Kaplan-Meier survival curves for the 14 differentially expressed long non-coding RNAs significantly associated with overall survival of glioblastoma multiforme patients. Kaplan-Meier survival curves for (A) AC005035.1, (B) AC010336.2, (C) AC022498.1, (D) AC108134.2, (E) AC116351.2, (F) Clorf132, (G) C10orf91, (H) DBH-AS1, (I) HOTAIR, (J) LINC00475, (K) MED4-AS1, (L) MIR210HG, (M) ZEB1-AS1 and (N) ZNF197-AS1. LINC00475, long intergenic non-protein coding RNA 475; AS1, antisense 1; DBH, dopamine $\beta$-hydroxylase; MED4, mediator complex subunit 4; MIR210HG, microRNA 210 host gene; ZEB1, zinc finger E-box binding homeobox 1; ZNF197, zinc finger protein 197.

A

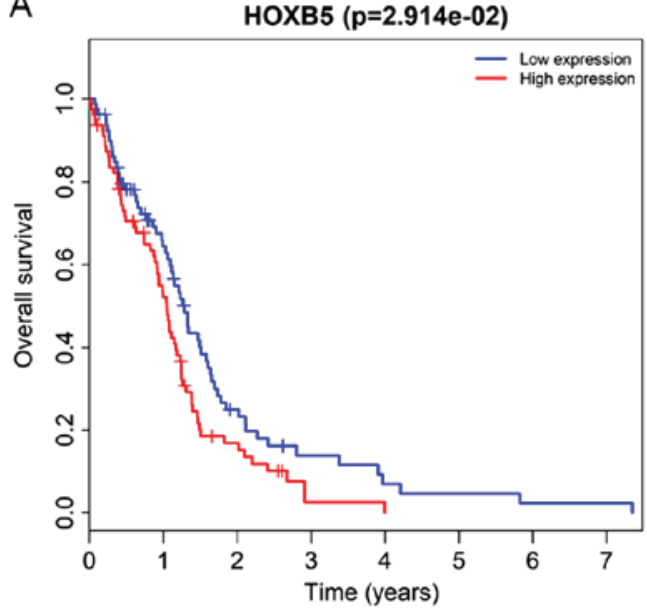

B

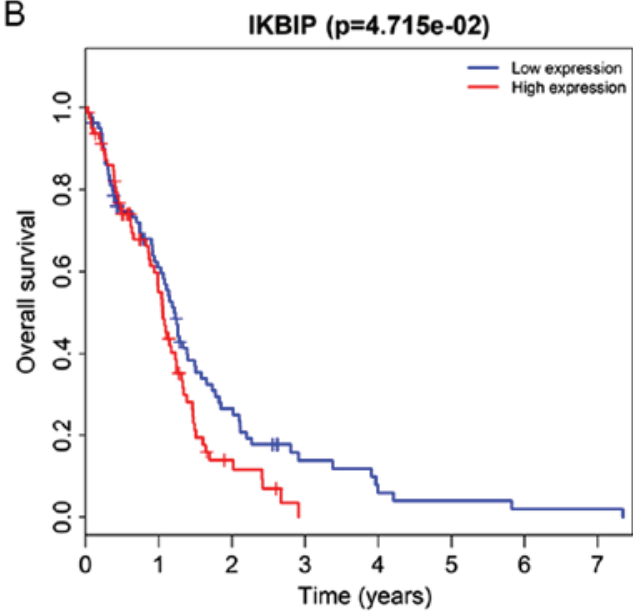

Figure 8. Kaplan-Meier survival curves for the 2 differentially expressed mRNAs significantly associated with overall survival of glioblastoma multiforme patients. Kaplan-Meier survival curves for (A) HOXB5 and (B) IKBIP. HOXB5, homeobox B5 IKBIP, inhibitor of NF-кB kinase subunit $\beta$-interacting protein. 


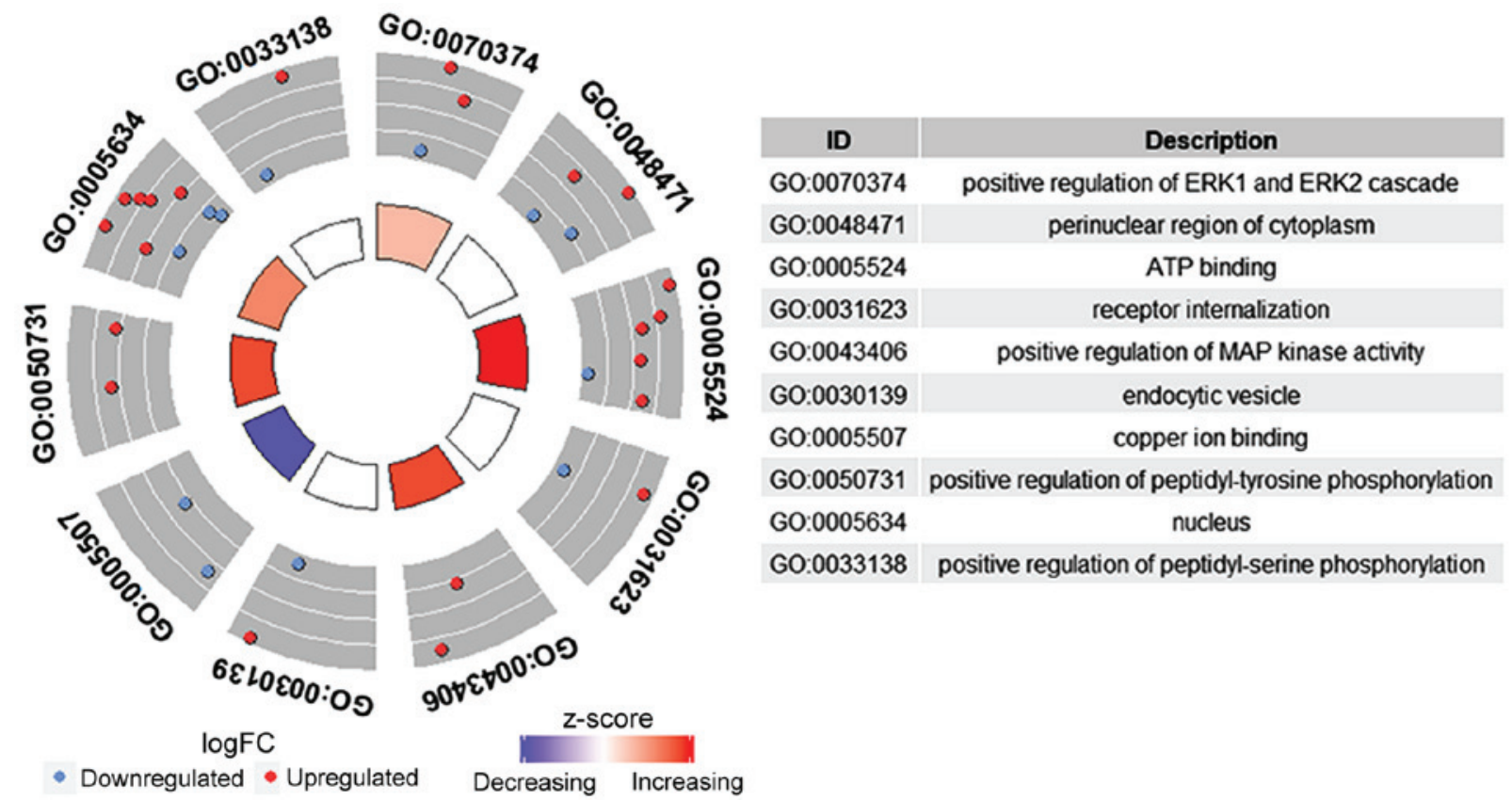

Figure 9. Predominant terms in the category Biological Process and all of the four terms in the categories Cellular Component and Molecular Function in the GO functional enrichment analysis. GO, Gene Ontology; FC, fold change.

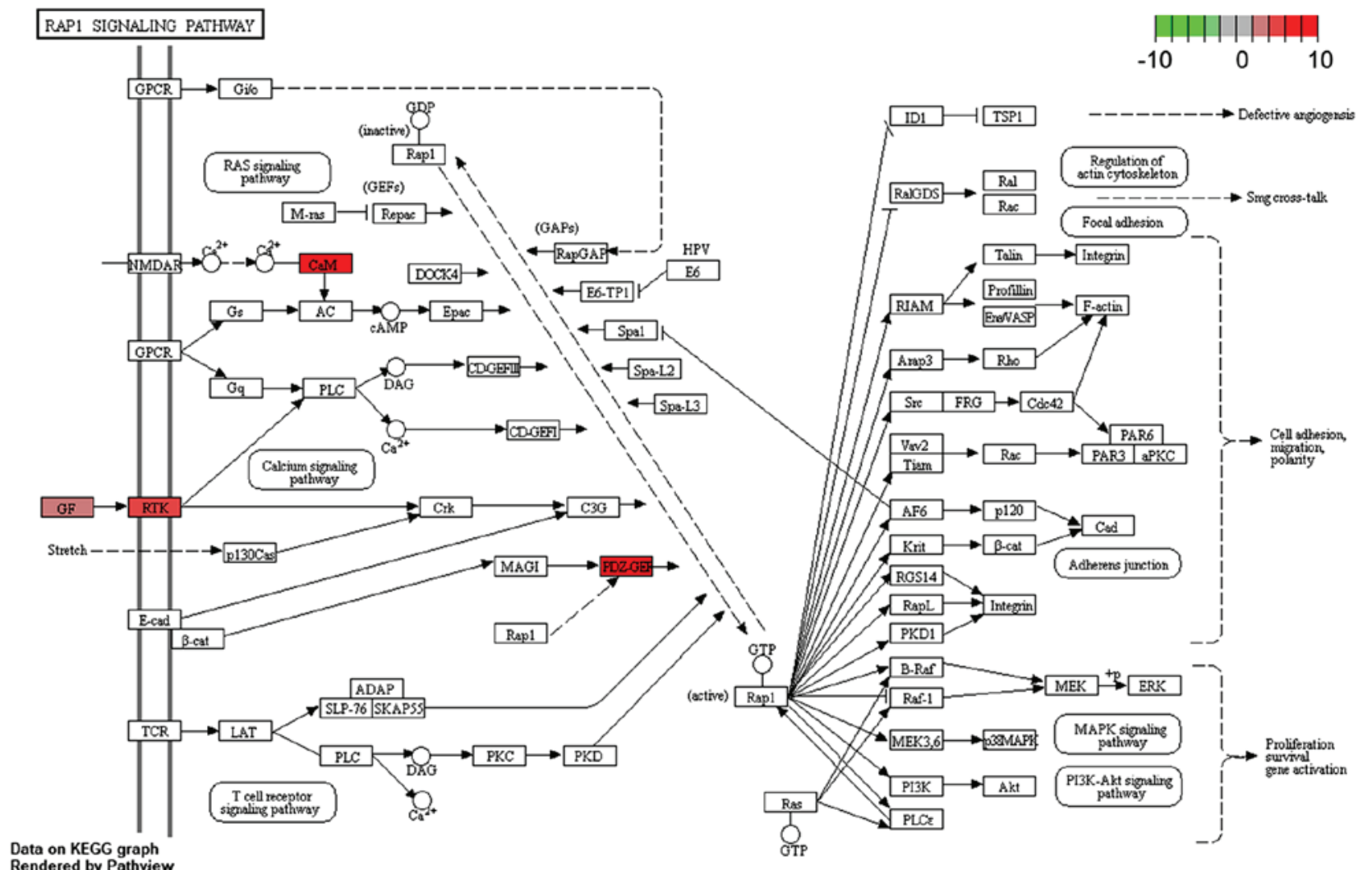

Figure 10. KEGG pathways enriched by the differentially expressed mRNAs in the competing endogenous RNA network. The Rap1 signaling pathway was indicated to be associated with the pathogenesis of glioblastoma multiforme. KEGG, Kyoto Encyclopedia of Genes and Genomes; Rap1, Ras-related protein 1.

to IncRNA and miRNAs, certain mRNAs also correlate with survival in GBM patients, including HOXB5 and inhibitor of nuclear factor $\kappa \mathrm{B}$ kinase-interacting protein (IKBIP). Patients with high expression levels of these two mRNAs have shorter survival times than those with low levels. HOXB5 has been reported to promote cell proliferation, migration and invasion in lung cancer, retinoblastoma and breast cancer (27-29). IKBIP is the target of tumor protein 53 with a pro-apoptotic function (30). To the best of our knowledge, the present study was the first to report the 
association of these two key mRNAs with the prognosis of GBM patients.

Functional enrichment analysis revealed that certain GO terms and pathways associated with transcriptional regulation and tumorigenesis, including the Rap1 signaling pathways, were enriched by the DEmRNAs. The close association between enriched KEGG pathways and the ceRNA network demonstrates the credibility of the results.

Of note, the present study had certain limitations. Due to the in silico nature of the study, there was a lack of experimental validation in vitro and in vivo. The present results and conclusions may serve as a foundation for the establishment of mechanistic hypotheses as a basis for further experiments on clinical samples and cell lines.

In conclusion, in the present study, GBM-associated lncRNAs, miRNAs and mRNAs were identified using cohorts from TCGA and GEO databases. A ceRNA network associated with lncRNAs was successfully constructed, providing perceptiveness into the newly proposed crosstalk among distinct types of RNA transcripts. A significant correlation between overall survival and clinical characteristics in patients with GBM may be established by analyzing key lncRNAs in future study. The present study enhances the understanding of the biological mechanisms of ceRNAs and helps to clarify the pathogenesis of GBM.

\section{Acknowledgements}

Not applicable.

\section{Funding}

The project was supported by the Science and Technology Project of Shenyang (grant no. 18-014-4-03) and the Science and Technology Project of the Education Department of Liaoning Province (grant no. LFWK201705).

\section{Availability of data and materials}

The datasets generated and/or analyzed during the current study are available from the corresponding author on reasonable request.

\section{Authors' contributions}

GL conceived and designed the study. SL performed the data mining, acquisition and analysis. GL and SL wrote and approved the final manuscript.

\section{Ethical approval and consent to participate}

Not applicable.

\section{Patient consent for publication}

Not applicable.

\section{Competing interests}

The authors declare that they have no competing interests.

\section{References}

1. Qi P and Du X: The long non-coding RNAs, a new cancer diagnostic and therapeutic gold mine. Mod Pathol 26: 155-165, 2013.

2. Amirkhah R, Schmitz U, Linnebacher M, Wolkenhauer O and Farazmand A: MicroRNA-mRNA interactions in colorectal cancer and their role in tumor progression. Genes Chromosomes Cancer 54: 129-141, 2015.

3. Wang $\mathrm{C}, \mathrm{Hu} \mathrm{DZ}$ and Liu JZ: Identification of critical TF-miRNA-mRNA regulation loops for colorectal cancer metastasis. Genetics Mol Res 14: 5485-5495, 2015.

4. Zhang C, Wang C, Jia Z, Tong W, Liu D, He C, Huang X and $\mathrm{Xu}$ W: Differentially expressed mRNAs, IncRNAs, and miRNAs with associated co-expression and ceRNA networks in ankylosin spondylitis. Oncotarget 8: 113543-113557, 2017.

5. Salmena L, Poliseno L, Tay Y, Kats L and Pandolfi PP: A ceRNA hypothesis: The Rosetta stone of a hidden RNA language? Cell 146: 353-358, 2011.

6. Liu K, Guo L, Guo Y, Zhou B, Li T, Yang H, Yin R and Xi T: AEG-1 3'-untranslated region functions as a ceRNA in inducing epithelial-mesenchymal transition of human non-small cell lung cancer by regulating miR-30a activity. Eur J Cell Biol 94: 22-31, 2015.

7. Peng W, Si S, Zhang Q, Li C, Zhao F, Wang F, Yu J and Ma R: Long non-coding RNA MEG3 functions as a competing endogenous RNA to regulate gastric cancer progression. J Exp Clin Cancer Res 34: 79, 2015.

8. Johnsson P, Ackley A, Vidarsdottir L, Lui WO, Corcoran M, Grandér D and Morris KV: A pseudogene long-noncoding-RNA network regulates PTEN transcription and translation in human cells. Nat Struct Mol Biol 20: 440-446, 2013.

9. Fan Q and Liu B: Comprehensive analysis of a long noncoding RNA-associated competing endogenous RNA network in colorectal cancer. Onco Targets Ther 11: 2453-2466, 2018.

10. Du Z, Fei T, Verhaak RG, Su Z, Zhang Y, Brown M, Chen Y and Liu XS: Integrative genomic analyses reveal clinically relevant long noncoding RNAs in human cancer. Nat Struct Mol Biol 20: 908-913, 2013.

11. Zhang W, Zhang J, Hoadley K, Kushwaha D, Ramakrishnan V, Li S, Kang C, You Y, Jiang C, Song SW, et al: MiR-181d: A predictive glioblastoma biomarker that downregulates MGMT expression. Neuro Oncol 14: 712-719, 2012.

12. Robinson MD, McCarthy DJ and Smyth GK: EdgeR: A bioconductor package for differential expression analysis of digital gene expression data. Bioinformatics 26: 139-140, 2010.

13. Jeggari A, Marks DS and Larsson E: MiRcode: A map of putative microRNA target sites in the long non-coding transcriptome. Bioinformatics 28: 2062-2063, 2012.

14. Agarwal V, Bell GW, Nam JW and Bartel DP: Predicting effective microRNA target sites in mammalian mRNAs. Elife 4: 2015.

15. Chou CH, Chang NW, Shrestha S, Hsu SD, Lin YL, Lee WH, Yang CD, Hong HC, Wei TY, Tu SJ, et al: MiRTarBase 2016: Updates to the experimentally validated miRNA-target interactions database. Nucleic Acids Res 44: D239- D247, 2016.

16. Wong $\mathrm{N}$ and Wang X: MiRDB: An online resource for microRNA target prediction and functional annotations. Nucleic Acids Res 43: D146-D152, 2015.

17. Dennis G Jr, Sherman BT, Hosack DA, Yang J, Gao W, Lane HC and Lempicki RA: DAVID: Database for annotation, visualization, and integrated discovery. Genome Biol 4: R60, 2003.

18. Yu G, Wang L-G, Han Y and He Q-Y: ClusterProfiler: An R package for comparing biological themes among gene clusters. OMICS 16: 284-287, 2012.

19. Roy S, Lahiri D, Maji T and Biswas J: Recurrent glioblastoma: Where we stand. South Asian J Cancer 4: 163-173, 2015.

20. Ebert MS, Neilson JR and Sharp PA: MicroRNA sponges: Competitive inhibitors of small RNAs in mammalian cells. Nat Methods 4: 721-726, 2007.

21. Brown BD, Cantore A, Annoni A, Sergi LS, Lombardo A, Della Valle P, D'Angelo A and Naldini L: A microRNA-regulated lentiviral vector mediates stable correction of hemophilia B mice. Blood 110: 4144-4152, 2007.

22. Zhang X, Zhang X, Hu S, Zheng M, Zhang J, Zhao J, Zhang X, Yan B, Jia L, Zhao J, et al: Identification of miRNA-7 by genome-wide analysis as a critical sensitizer for TRAIL-induced apoptosis in glioblastoma cells. Nucleic Acids Res 45: 5930-5944, 2017. 
23. Yu J, Cai X, He J, Zhao W, Wang Q and Liu B: Microarray-based analysis of gene regulation by transcription factors and microRNAs in glioma. Neurol Sci 34: 1283-1289, 2013.

24. Liang F, Yang M, Tong N, Fang J, Pan Y, Li J and Zhang X: Identification of six key miRNAs associated with breast cancer through screening large-scale microarray data. Oncol Lett 16: 4159-4168, 2018.

25. Li G, Huang M, Cai Y, Yang Y, Sun X and Ke Y: Circ-U2AF1 promotes human glioma via derepressing neuro-oncological ventral antigen 2 by sponging hsa-miR-7-5p. J Cellular Physiol 234: 9144-9155, 2019.

26. Cao Y, Wang P, Ning S, Xiao W, Xiao B and Li X: Identification of prognostic biomarkers in glioblastoma using a long non-coding RNA-mediated, competitive endogenous RNA network. Oncotarget 5: 41737-41747, 2016.

27. Lee JY, Kim JM, Jeong DS and Kim MH: Transcriptional activation of EGFR by HOXB5 and its role in breast cancer cell invasion. Biochem Biophys Res Commun 18: 2924-2930, 2018.
28. $\mathrm{Xu} \mathrm{H}$, Zhao $\mathrm{H}$ and $\mathrm{Yu}$ J: HOXB5 promotes retinoblastoma cell migration and invasion via ERK1/2 pathway-mediated MMPs production. Am J Transl Res 15: 1703-1712, 2018.

29. Zhang B, Li N and Zhang H: Knockdown of homeobox B5 (HOXB5) inhibits cell proliferation, migration, and invasion in non-small cell lung cancer cells through inactivation of the Wnt/ $\beta$-catenin pathway. Oncol Res 19: 37-44, 2018.

30. Hofer-Warbinek R, Schmid JA, Mayer H, Winsauer G, Orel L, Mueller B, Wiesner CH, Binder BR and de Martin R: A highly conserved proapoptotic gene, IKIP, located next to the APAF1 gene locus, is regulated by p53. Cell Death Differ 11: 1317-1325, 2004.

cc) (i) () $\odot$ This work is licensed under a Creative Commons c) Attribution-NonCommercial-NoDerivatives 4.0 International (CC BY-NC-ND 4.0) License. 